PLANETARY FIREWORKS

\section{The Comet Shoemaker-Levy 9 Hits Jupiter This Summer}

Willem Wamsteker who manages the ESA-International Ultraviolet Explorer Observatory at the ESA Satellite Tracking Centre, Madrid, explains why coordinated, world-wide observations of the impact of a comet with Jupiter will contribute enormously to our understanding of planetary phenomena.

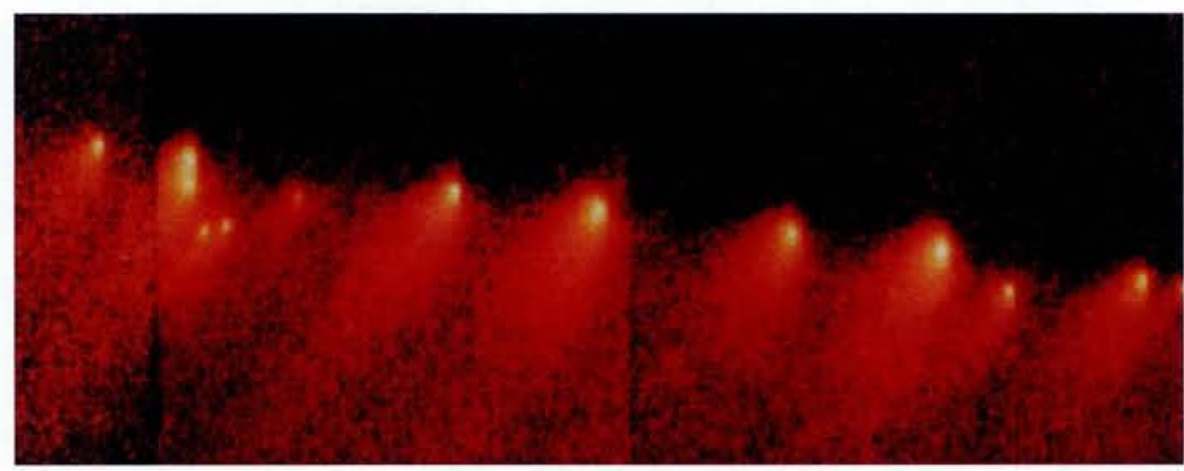

Fig. 1 - A composite image taken in January 1994 with the Hubble Space Telescope's refurbished Wide-Field Planetary Camera of the "string of pearls" that used to be the comet Shoemaker-Levy 9. Some 13 of the 21 pieces into which the comet has disintegrated can be identified (ESA/NASA photograph).

Most excitement in astronomy and planetary sciences is usually associated with unpredictable phenomena or events involving long lead-times such as supernovae explosion (e.g., SN 1987A) and perihelion passages of periodic comets (e.g., the 1985/86 passage of the comet P/Halley). These occasions give rise to frantic observational activities which in the long run, after the "news" value has evapourated, generally lead to a major enhancement in our understanding of the physics involved. They generally imply initiatives which do not require too much coordination. Comet P/Halley was an exception since all major space agencies were active, together with complex space experiments such as ESA's GIOTTO mission when the spacecraft flew within $596 \mathrm{~km}$ of the comet in March 1986.

No one could foresee that the discovery of the comet Shoemaker-Levy 9 (SL-9) in March 1993 would present us with one of the more exciting predictable phenomena. SL-9 is special because during its last passage past Jupiter on 8 July 1992 at a distance of some 1.3 Jovian radii, this loosely bound 5-10 km-sized package of "dirty ice" suffered sufficient gravitational torque from Jupiter to break it up in what is now referred to as a "string of pearls" - a set of smaller bodies (estimated sizes of up to $1 \mathrm{~km}$ ) which follow in a nice sequence the orbit of the comet. The break-up of comets is not unusual and has been observed on several occasions; in the case of SL-9 it suggests a primary body of low density and with a tensile strength equal to the value normally associated with cometary nuclei.

It was soon realized that all the 21 identified pieces into which SL-9 has split up will collide with Jupiter between July 17 and July 21, 1994. The impact will take place close to the Jovian morning terminator, hidden from direct viewing from the Earth. Although a visible impact on the front of the planet would obviously be more spectacular, the current
Further information obtainable from:

- the ESA coordinator for SL-9 activities [F. Billebaud: fran@altair.univ-lyon1.fr];

- the bulletin board of the NASA coordinator [Dr. M. A'Hearn: pdssbn.astro.umd.edu with the password c1993e].

The large body of information collected by $\mathrm{G}$. Orton, the Atmospheres Chairman of the International Jupiter Watch, is available by anonymous ftp at lono.jpl.nasa.gov in the subdirectory /pub/ijwnotices, where the information notices are coded by date as goyymmdd.

because a collision of a cometary with a planetary atmosphere will allow completely new phenomena to be studied to yield important data about aspects of atmospheric physics which are otherwise impossible to obtain. The effects of such impact can be appreciated if one recognizes that a $100 \mathrm{~m}$ piece of comet at an approach velocity of some $14 \mathrm{~km} / \mathrm{s}$ was involved in forming the huge Tunguska crater in 1908. In the case of SL-9 we are looking at the impact of $\mathrm{km}$ sized chunks impacting at a velocity of about $60 \mathrm{~km} / \mathrm{s}$. An enormous observational effort is needed since many physical phenomena are involved in the impact (see insert). The programme under preparation involves most scientific spacecraft currently operating while all major ground-based observatories will dedicate considerable amounts of observing time to the event using many different modes (direct imaging, spectroscopy extending from the ultraviolet to the far-infrared, radio observations, etc.). Owing to the large variety of phenomena to be studied, each of the observations is expected to contribute in an important way to the overall effort. The effort must be world-wide if one wants to observe all the impacts. The only facilities capable of making continuous observations throughout the impact are the GALILEO and the International Ultraviolet Explorer (IUE) spacecraft. As the Earth's weather is unpredictable, amateur astronomers are expected to play an important rôle.

An interesting coincidence is that a similar coordination programme was mounted, albeit on a smaller scale, during the second World

The prediction of impacts triggered a flurr of activities at some 40 observatories, mainly

\section{SL-9 Impact Phenomena}

A variety of impact phenomena are involved in the impact of SL-9 with Jupiter. They include:

- Exposure of the interior of the comet. This material has probably not been exposed to direct irradiation since the formation of the solar system itself.

- Magnetospheric disturbances in the very active Jovian dipole field and the associated disruptions of the field-aligned currents.

- The distribution of highly charged dust after entry of the "string of pearls" into the Jovian magnetosphere.

- Atmospheric photo-ion chemistry in the upper Jovian atmosphere when magnetic fields are disrupted; upper-level winds with dust transport.

- Modification of the carbon chemistry in the upper atmosphere of Jupiter, especially when materials normally confined to the deep atmosphere, such as $\mathrm{SiH}_{4}, \mathrm{H}_{2} \mathrm{~S}, \mathrm{H}_{2} \mathrm{O}$, and $\mathrm{HCN}$, are transferred to the upper troposphere and stratosphere.

- Shocks and explosive events ("fireballs") when the comet nuclei enter supersonically the increasingly dense layers of the lower Jovian atmosphere, where nuclei of different sizes are expected to explode at different levels (penetration down to the cloud layer at 1-10 bar for the largest segments, while a $100 \mathrm{~m}$ fragment is not expected to penetrate beyond the 0.1 bar level).

- The direct effects of the explosive destruction of the comet nuclei will release energy at a rate of $10^{25}-10^{30} \mathrm{ergs} / \mathrm{s}$ compared to a total solar input on Jupiter of $10^{26} \mathrm{ergs} / \mathrm{s}$. This energy release may excite larger amplitudes in Jupiter's normal-mode oscillations. 
Astronomy Day in 1993 within the context of the International Space Year. The data that were collected will supply an importance reference set for the observations to be made this July during the impacts.

The possibility exists that the phenomena will not live up to the public's expectations for we cannot expect astrophysical forecasting to be as accurate as weather forecasts, even in these times of advanced techniques and extensive whole-Earth observation from satellites. However, we can be sure that the observational effort will contribute enormously to our understanding of planetary phenomena, especially to atmospheric physics and the interactions between particles in planetary magnetospheres.
Fig. 2 - A drawing (not to scale) illustrating the geometry of the collision between the comet SL-9 and the planet Jupiter. A wide variety of plasma and atmospheric phenomena will take place in the very dynamic environment of the Jovian system. (Courtesy of $R$. Prange, IAS, Orsay)

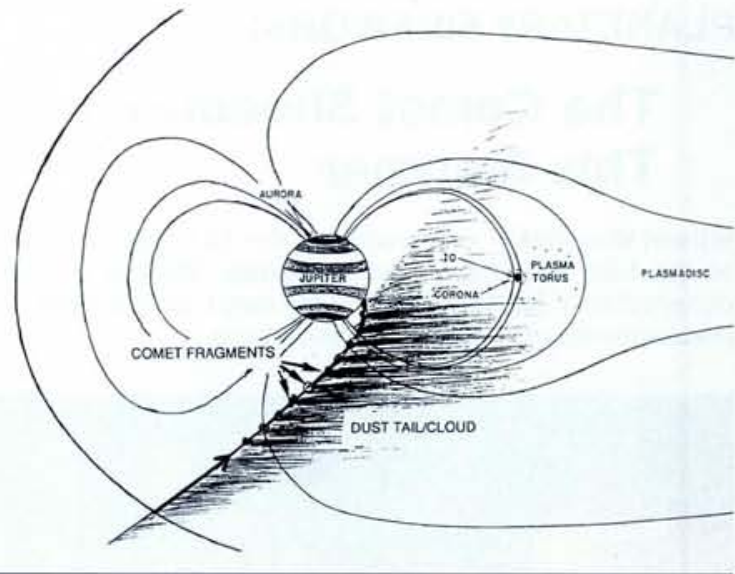

UNK, Protvino

\section{A Milestone in Spite of Hardships}

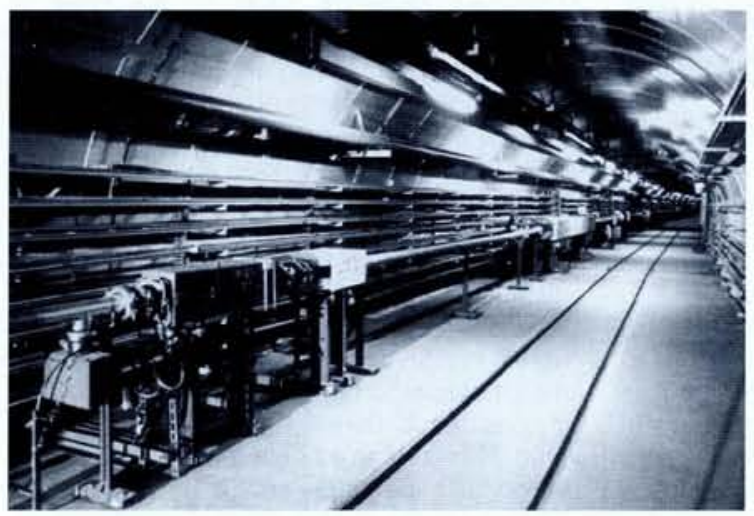

View of the UNK's transfer-line tunnel showing the beam-line magnets.

A typical control screen for the transfer line. At the top, a synoptic view of equipment in the beam line; at the centre, so-called "sliders" allowing adjustment of magnet currents; at the bottom, horizontal and vertical beam profiles as measured at points along the line. The control software was developed using Vsystem, a commercial controls package from Vista Control Systems, Inc., Los Alamos, USA.

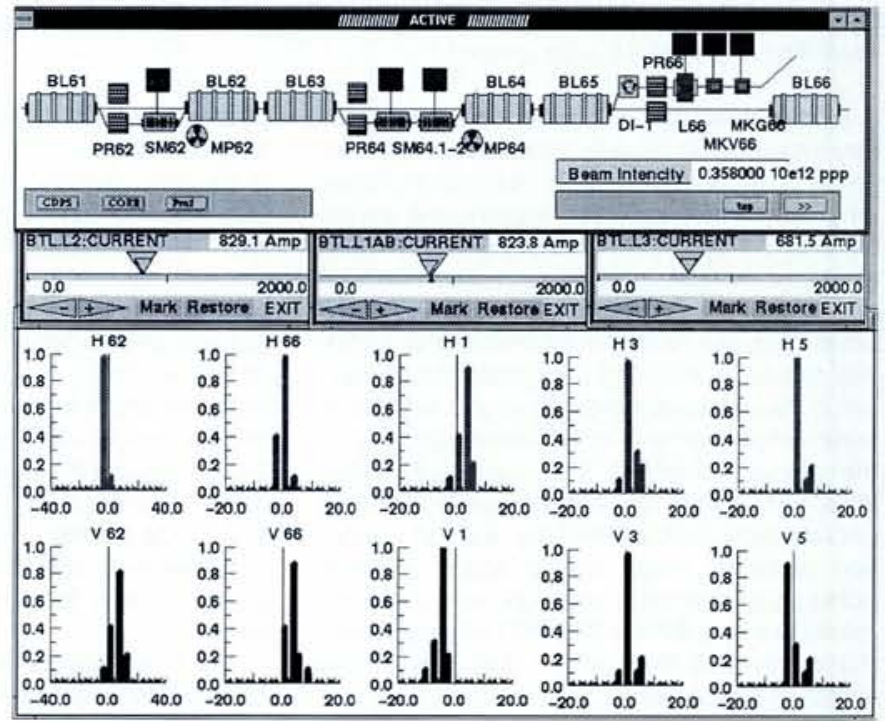

design value. The combination of beam diagnostics and control systems, which were in part developed in collaboration with CERN, allowed operation of the whole beam-transfer line from one central point in a user-friendly way.

Accelerator staff and particle physicists everywhere rejoice with their Russian colleagues in Protvino and hope that the milestone signifies a lasting positive turn in the UNK endeavour.

B. Kuiper, CERN that this transfer line be tried out with an actual proton beam well in advance of the accelerator itself, as was the case for CERN's LEP collider.

The exercise aimed to test two main aspects: first, the functioning of the design and the implementation of the beam ejection and transfer line and, second, the integration of the equipment into a coherent unit using the beam diagnostics and control systems. The $2.7 \mathrm{~km}$-long transfer line houses 52 dipole magnets of $5.8 \mathrm{~m}$ in length, 88 quadrupole lenses and 56 correction magnets. The beam diagnostics comprise 3 current monitors, 46 position monitors, 26 profile monitors as well as beam loss and halo monitors. The control system uses microprocessor-based equipment controllers, personal computers at an intermediate level and, as the operator interface, workstations with modern graphics software packages.

The injector synchrotron U70 accelerated 5 out of the possible 30 proton bunches at $6 \mathrm{MHz}$ to $65 \mathrm{GeV}$, at which energy the beam was recaptured at $200 \mathrm{MHz}$, as is required for UNK. A beam of about $3.5 \mathrm{x}$ $10^{11}$ protons per pulse was thus ejected into the transfer line and successfully steered up to a beam stopper placed at $2 \mathrm{~km}$ from the start. The emitance measured at that point was in good agreement with the

\section{Conference on EMERGENCE OF MODERN PHYSICS} Berlin, 20-24 March 1994

Organized by:

- History of Physics Division of the German Physical Society

- EPS Interdivisional Group on the History of Physics

- Commission on the History of Modern Physics of the IUHPS-

DHS

In conjunction with the celebration of the 150th Anniversary of the German Physical Society

To commemorate the centenary of the discovery of X-rays, the Zeeman effect, radioactivity, and the electron and to analyze the accompanying debates that shaped modern physics.

\section{Abstracts by 1 September 1994}

Contact: Fabio Bevilacqua, Dip. di Fisica, Univ. di Pavia,

Via Bassi, 6, I-27100 Pavia; tel./fax: +39-382-50 74 95 / 507563 